\title{
Fault Diagnosis of the Gyratory Crusher Based on Fast Entropy Multilevel Variational Mode Decomposition
}

\author{
Fengbiao Wu $\mathbb{D},{ }^{1,2}$ Lifeng Ma $\mathbb{D}^{1},{ }^{1}$ Qianqian Zhang, ${ }^{3}$ Guanghui Zhao, ${ }^{1}$ and Pengtao Liu ${ }^{1}$ \\ ${ }^{1}$ College of Mechanical Engineering, Taiyuan University of Science and Technology, Taiyuan 030024, China \\ ${ }^{2}$ Shanxi Institute of Energy, Taiyuan 030006, China \\ ${ }^{3}$ School of Automation and Software, Shanxi University, Taiyuan, Shanxi 030006, China \\ Correspondence should be addressed to Lifeng Ma; mlf_zgtyust@163.com
}

Received 22 April 2021; Accepted 15 June 2021; Published 25 June 2021

Academic Editor: Liang Guo

Copyright $\odot 2021$ Fengbiao Wu et al. This is an open access article distributed under the Creative Commons Attribution License, which permits unrestricted use, distribution, and reproduction in any medium, provided the original work is properly cited.

Gyratory crusher is a kind of commonly used mining machinery. Because of its heavy workload and complex working environment, it is prone to failure and low reliability. In order to solve this problem, this paper proposes a fault diagnosis method of the gyratory crusher based on fast entropy multistage VMD, which is used to quickly and accurately find the possible fault problems of the gyratory crusher. This method mainly extracts the vibration signal by combining fast entropy and variational mode decomposition, so as to analyze the components of the vibration signal. Among them, fast entropy is used to quickly determine the number of modes in the signal spectrum and the bandwidth occupied by the modes. The extracted parameters can be converted into the input parameters of VMD. VMD can accurately extract the modal components in the signal by inputting the number of modes and related parameters. Due to the differences between modes, using the same parameters to extract the modes often leads to inaccurate results. Therefore, the concept of multilevel VMD is proposed. The parameters of different modes are determined by fast entropy. The modes in the signals are separated and extracted with different parameters so that different signal modes can be accurately extracted. In order to verify the accuracy of the method, this paper uses the data collected from the rotary crusher to test, and the results show that the proposed FE method can quickly and effectively extract the fault components in the vibration signal.

\section{Introduction}

Gear and rolling bearing are widely used parts in various rotating machinery, and they are important structures carrying transmission and force transmission in rotating machinery. Therefore, in recent years, the research on safety and reliability of rotating parts has been highly concerned, and the related research fields include mechanical fault diagnosis, life prediction, and health operation and maintenance [1]. In all kinds of mechanical faults, the mechanical fault accidents caused by gear components or rolling bearings account for more than $70 \%$. Therefore, it is of great significance to study and find the fault problems existing in the operation of gear components or rolling bearings in time for improving the safety and reliability of the mechanical system [2]. There are many methods to study the faults of rotating mechanical parts. The most widely used methods in academic circles are to collect the vibration signals generated by the components under stable working conditions. The fault conditions of mechanical components are evaluated by analyzing the composition of vibration signals [3, 4]. At present, there are two main methods to analyze vibration signals: one is to analyze the components of signals through the time-frequency information of signals [5], and the other is to decompose the signals into different modal components by the decomposition algorithm $[6,7]$. Among them, the methods to obtain the frequency band components by analyzing the spectrum include the fast kurtosis spectrum proposed by Antoni [8] and the fast entropy method proposed by Zhang et al. [9]. Signal mode decomposition is also a common method type in the field of fault diagnosis. For example, Fei used the combination of the wavelet transform 
and relevance vector machine to verify the role of wavelet transform in signal analysis [10] and also used the improved algorithm based on empirical mode decomposition for rolling bearing fault diagnosis $[11,12]$. At present, the most commonly used and ideal mode decomposition method is variational mode decomposition. There are many applications of variational mode decomposition. He et al. combined the variational mode decomposition method with the neural network for intelligent diagnosis of the wind turbine rotating fault [13], and Zhao et al. proposed combining variational mode decomposition and signal spectrum entropy to determine the weak fault component of rotating machinery vibration signal methods [14].

The above methods have advantages in various applications, but for the rotating machinery with complex working conditions, the time-frequency analysis method has many characteristic parameters, so it is often unable to extract the main fault features of the signal effectively [15]. For the method of variational mode decomposition, because the components of the vibration signal are complex, using this kind of method for component analysis easily causes the problems of signal mode aliasing and mode underdecomposition or overdecomposition [16]. In order to solve the problem of fault diagnosis of rotating machinery, such as mining machinery or high-strength machinery assembly line, this paper proposes a multilevel VMD method based on fast entropy by combining the entropy spectrum and variational mode decomposition. For the vibration signal with complex components, the direct use of variational mode decomposition is unable to effectively extract various fault components in the signal [17]. Fast entropy can quickly extract the principal components of the signal spectrum and provide calculation parameters for each level of VMD. Multilevel VMD solves the problem of single-parameter mismatch through multiple-mode extraction of the signal. At the same time, multilevel VMD can further extract the weak impact components of the signal. The proposed fast entropy multilevel VMD method has high efficiency of feature extraction. It has the advantage of accurate feature data. Therefore, firstly, the entropy spectrum method is used to determine the modal components in the signal as VMD method extraction parameters [18]. In order to prevent the loss of effective components, the decomposed modal components are separated from the signal, and the remaining signals are extracted again until the decomposition termination parameters calculated by the entropy spectrum are met; thus, the effective components in the signal are decomposed [19] to solve the problem that the signal component is not easy to determine under complex conditions. In order to further verify the application ability of the proposed method, it is necessary to classify the extracted components to judge the ability of the proposed method in the fault diagnosis of rotating machinery [20]. Modal components can be classified by various classification methods. The common fault classifier methods include SVM (support vector machine) linear classifier, datadriven, convolutional neural network, and decision tree model [21-24]. In this paper, a classification method of decision tree model, XGBoost, is used as a classifier. XGBoost has the characteristics of fast discrimination speed and good classification performance for the twodimensional data, so it is suitable for the classification of modal components in this paper [25].

In the second part of this paper, the theory of fast entropy and variational mode decomposition is introduced. In the third part, the principle of the improved method combining fast entropy and VMD is introduced in detail. In the experimental part, the proposed method is verified by the actual vibration signal collected from the experimental platform, and the method is analyzed and summarized at the end of the section.

\section{Principles}

The improved method proposed in this paper mainly involves two kinds of vibration signal analysis methods, which are variational mode decomposition (VMD) and fast entropy spectrum (FE). Among them, the variational mode decomposition method is a classical signal decomposition method which iteratively decomposes the vibration signal into several eigenmodes [26], and the fast entropy spectrum is a method used to determine the number of signal modes through the signal spectrum [7]. These two methods are described in detail in this section.

2.1. Variational Mode Decomposition. Variational mode decomposition is an adaptive mode decomposition method, which is widely used in the modal decomposition of vibration signals. Given the number of modes, VMD can obtain the best eigenmode and band center frequency through iterative optimization. Compared with the traditional EMD, wavelet transform, and other analysis methods, VMD has higher efficiency and decomposition accuracy, which is a very important method in the field of signal analysis. The variational mode decomposition is to extract the vibration modes by iteratively solving the vibration signals as variational functions:

$$
\min _{\left\{u_{k}\right\},\left\{\omega_{k}\right\}}\left\{\sum_{k}\left\|\partial_{t}\left[\left(\frac{\delta(t)+j}{\pi t}\right) * u_{k}(t) e^{-j \omega_{k} t}\right]\right\|_{2}^{2}\right\} .
$$

The signal $f$ can be obtained by superposition of $K$ modes:

$$
\text { s.t. } \sum_{k=1}^{K} u_{k}=f \text {. }
$$

In formulas (1) and (2), $K$ is the number of modes, $\left\{u_{k}\right\}$ is the $k$ th modal component after decomposition, $\left\{\omega_{k}\right\}$ is the center frequency of the corresponding modal component, $\delta(t)$ is the Dirac function, and $*$ is the convolution operator.

The Lagrange multiplication operator is introduced to transform the constrained variational problem into an unconstrained variational problem: 


$$
L\left(\left\{u_{k}\right\},\left\{\omega_{k}\right\}, \lambda\right)=\alpha \sum_{k}\left\|\partial_{t}\left[\left(\frac{\delta(t)+j}{\pi t}\right) * u_{k}(t) e^{-j \omega_{k} t}\right]\right\|_{2}^{2}+\left\|f(t)-\sum_{k} u_{k}(t)\right\|_{2}^{2}+\left\langle\lambda(t), f(t)-\sum_{k} u_{k}(t)\right\rangle .
$$

In the formula, the quadratic penalty term is introduced, which can be used to reduce the noise interference in the signal, then the alternating direction multiplier iterative algorithm (ADMI) is used to optimize the overlapping band, and the optimal mode and its center frequency are obtained.

The mode is optimized iteratively:

$$
\frac{\widehat{f}(\omega)-\sum_{i \neq k} \widehat{u}_{i}(\omega)+\hat{\lambda}(\omega) / 2}{1+2 \alpha\left(\omega-\omega_{k}\right)^{2}} \longrightarrow \widehat{u}_{k}^{n+1}(\omega) .
$$

The center frequency is optimized iteratively:

$$
\frac{\int_{0}^{\infty} \omega\left|\widehat{u}_{k}^{n+1}(\omega)\right|^{2} \mathrm{~d} \omega}{\int_{0}^{\infty}\left|\widehat{u}_{k}^{n+1}(\omega)\right|^{2} \mathrm{~d} \omega} \longrightarrow \omega_{k}^{n+1}(\omega) .
$$
follows:

The iterative process of the Lagrange multiplier is as

$$
\hat{\lambda}^{n}(\omega)+\gamma\left(\hat{f}(\omega)-\sum_{k} \widehat{u}_{k}^{n+1}(\omega)\right) \longrightarrow \widehat{\lambda}^{n+1}(\omega) .
$$

In equation (6), noise tolerance $\lambda$ is introduced for signal fidelity. The variable mode decomposition sets the maximum iteration times and the mode output conditions given the number of modes and the secondary penalty term and finally decomposes the signal into $K$ eigenmodes which reflect the main information of the vibration signal.

2.2. Fast Entropy. Entropy reflects the internal energy transformation of the signal. The impact components in the signal spectrum can be judged by entropy. Envelope entropy can screen the impact components in the signal spectrum, but its accuracy is not high, and it is greatly affected by noise in the actual complex working conditions. Fast entropy predicts the change of the impact components in the spectrum. The noise factor can be screened out to a certain extent. Fast entropy spectrum method is an improved method of fast kurtosis spectrum. Kurtosis spectrum is a computing tool used to detect nonstationary factors in signals. Kurtosis, as a parameter reflecting the change of the signal, can be used to detect the abnormal components in the stationary vibration signal, but this method has poor effect in the case of strong noise. In order to overcome this defect, the concept of kurtosis spectrum is proposed to overcome the difficulty of using kurtosis to determine the components of the strong noise signal. The spectrum is divided into equal scales until the signal spectrum is divided into two parts. The frequency band of each mode is included to determine the modal component of the signal. Fast kurtosis spectrum is a method that combines the kurtosis spectrum with the FIR filter. Fast entropy is a method that divides the signal spectrum through the trend spectrum on the basis of the fast kurtosis spectrum, so as to more accurately divide the signal mode. The basic principle of fast entropy is as follows.

Firstly, the signal spectrum is calculated by short-time Fourier transform. The spectrum $\widehat{f}(\omega)$ of signal $f(t)$ is obtained by Fourier transform, and the key function $|\widehat{g}(u)|$ is obtained by discretizing the signal:

$$
\begin{aligned}
\widehat{f}(\omega) & =\int_{-\infty}^{+\infty} f(t) e^{-i \omega t} \mathrm{~d} t, \\
\hat{g}(u) & =\sum_{n=0}^{L-1} g(n) e^{-i(2 \pi / L) u n} .
\end{aligned}
$$

The trend spectrum of the signal is calculated:

$$
T(f)=\int_{-\infty}^{+\infty} \widehat{g}_{p}(u) e^{i 2 \pi u f} \mathrm{~d} u .
$$

In the fast entropy spectrum method, after the signal is divided through the trend spectrum, the components of the divided spectrum are extracted through the frequency slice function:

$$
W_{f}(t, \omega, \lambda, \sigma)=\frac{1}{2 \pi} \lambda \int_{-\infty}^{+\infty} \hat{f}(u) \widehat{p}^{*}\left(\frac{u-\omega}{\sigma}\right) e^{i u t} \mathrm{~d} u,
$$

where $t, \omega$, and $u$ are the observation time, observation frequency, and evaluation rate, scale factor $\sigma \neq 0$, parameter $\lambda$ is a constant, $\widehat{p}(\omega)$ represents the frequency slice function, and $*$ is conjugate. The time domain of the frequency-sliced wavelet transform can be expressed as

$$
W_{f}(t, \omega, \sigma)=\sigma e^{i \omega t} p^{*}(\sigma(\tau-t)) \mathrm{d} \tau .
$$

When $p(t)$ and $\widehat{p}(\omega)$ take special values, the frequency slice wavelet transform will be transformed into the traditional short-time Fourier transform, so it shows that the method is feasible in the generalized range.

Let $\sigma \propto \omega$ and $\kappa=\sigma / \omega$; the results are as follows:

$$
W_{f}(t, \omega, \kappa)=\frac{1}{2 \pi} \int_{-\infty}^{+\infty} \hat{f}(u) \widehat{p}^{*}\left(k \frac{u-\omega}{\omega}\right) e^{i u t} \mathrm{~d} u .
$$

$\kappa$ is defined as the relative resolution related to observation frequency and evaluation frequency. By substituting $\kappa,(10)$ can be transformed into the following forms:

$$
W_{f}(t, \omega, \kappa)=\frac{1}{k} \omega e^{i \omega t} \int_{-\infty}^{+\infty} f(\tau) e^{-i \omega t} p^{*}\left(\frac{\omega(\tau-t)}{k}\right) \mathrm{d} \tau .
$$

$\Delta \omega_{p}$ is the frequency window width of the frequency wavelet. Considering the bandwidth-frequency ratio of the 
frequency slice function, the frequency resolution of the frequency slice wavelet transform is set as $\eta_{p}=\sigma \Delta \omega_{p} / \omega=\Delta \omega_{p} / \kappa$. The frequency resolution of the signal is set to $\eta_{p}=\Delta \omega_{s} / \omega_{s}$. In general, $\eta_{p} \ll 1$. Therefore, $\eta_{p}$ can be achieved by adjusting $\kappa$. If $\widehat{p}(\omega)$ satisfies $\widehat{p}(0)=1$, the components of the original signal can be reconstructed by the following formula:

$$
f(t)=\frac{1}{2 \pi} \int_{-\infty}^{+\infty} \int_{-\infty}^{+\infty} W_{f}(\tau, \omega, \kappa) e^{i \omega(t-\tau)} \mathrm{d} \tau \mathrm{d} \omega
$$

2.3. XGBoost. Gradient surge decision tree (XGBoost) is a kind of decision tree method which can realize the rapid classification of samples. The process of constructing the XGBoost model is as follows: firstly, the modal components obtained by decomposition are used as samples. follows:

For a single sample $X_{m, y}$, the prediction results are as

$$
\begin{aligned}
\widehat{y}_{X} & =\varnothing\left(X_{m, y}\right) \\
& =\sum_{m=1}^{M} \eta_{m} f_{m}\left(X_{m, y}\right) .
\end{aligned}
$$

$\widehat{y}_{X}$ is the prediction result of the decision tree, $X_{m, y}$ denotes a sample with $m$ characteristic classes of $y$, and $f_{m}$ is the tree model of the $m$ th tree. Through XGBoost training, each tree can obtain the corresponding weight value $w$ and the tree structure parameter $q$ according to feature learning. In addition to the weight of the tree model obtained through training, this method increases the weight value of feature $\eta_{m}$ to modify the results of each tree model, so as to improve the accuracy of the results of the model.

The objective function of the XGBoost decision tree is

$$
L_{m}(\varnothing)=\sum_{i} l\left(\hat{y}_{i}, y_{i}\right)+\sum_{m} \Omega\left(f_{m}\right)
$$

where $l$ is the loss function of the model tree, which is used to reduce the error between the predicted value and the real value and form the basic tree model structure, and $\Omega$ is the regular term of the model tree, which is used to control the complexity of the tree model so that the learner can avoid overfitting as much as possible.

The objective function is solved. Firstly, the loss function is expanded to the second order by Taylor expansion:

$$
\mathrm{Obj}=\sum_{i=1}^{n}\left(l\left(y_{i}, \hat{y}_{i}^{(t-1)}\right)+g_{i} f_{t}\left(x_{i}\right)+\frac{1}{2} h_{i} f_{t}^{2}\left(x_{i}\right)\right)+\Omega\left(f_{t}\right)+\text { constant }
$$

The tree structure is defined:

$f_{t}(x)=\omega_{q(x)}, \quad \omega \in R^{T}, q: R^{d} \longrightarrow\{1,2, \ldots, T\}$.

The complexity of a tree is defined:

$$
\Omega\left(f_{t}\right)=\gamma T+\frac{1}{2} \lambda \sum_{j=1}^{T} \omega_{j}^{2}
$$

The final objective function is as follows:

$$
\text { Obj }=\sum_{j=1}^{T}\left[G_{j} \omega_{j}+\frac{1}{2}\left(H_{j}+\lambda\right) \omega_{j}^{2}\right]+\Upsilon T .
$$

Best of point:

$$
\begin{gathered}
\omega_{j}^{*}=-\frac{G_{j}}{H_{j}+\lambda}, \\
\mathrm{Obj}=-\frac{1}{2} \sum_{j=1}^{T} \frac{G_{j}^{2}}{H_{j}+\lambda}+\Upsilon T .
\end{gathered}
$$

The basis of the leaf node division is as follows:

gain $=\frac{1}{2}\left[\frac{G_{L}^{2}}{H_{L}+\lambda}+\frac{G_{R}^{2}}{H_{R}+\lambda}-\frac{\left(G_{L}+G_{R}\right)^{2}}{H_{L}+H_{R}+\lambda}\right]-\Upsilon$.

\section{The Improved Method Is Put Forward}

Because the decomposition performance of VMD depends on the selection of parameters, the number of modes and the selection of secondary penalty factors have great influence on the decomposition results. In the previous research of the VMD method, most scholars improved the performance of VMD mainly reflected in the optimization of parameters. However, in the actual signal, the number of modes in the signal is not easy to determine due to the existence of noise and environmental noise generated by the mechanical system. In addition, the frequency band width of different modes in the spectrum is not consistent due to the difference of spectrum characteristics of different modes. At present, most of the signal decomposition methods do not notice this key point. Therefore, this paper proposes a method of multistage variational mode decomposition (FE-MVMD) based on fast entropy. By using fast entropy, the parameters required by different series of VMD are obtained continuously to adapt the corresponding parameters of different modes of extraction, aiming to further improve the effect of the decomposition.

The flowchart of the proposed improvement method is shown in Figure 1. 


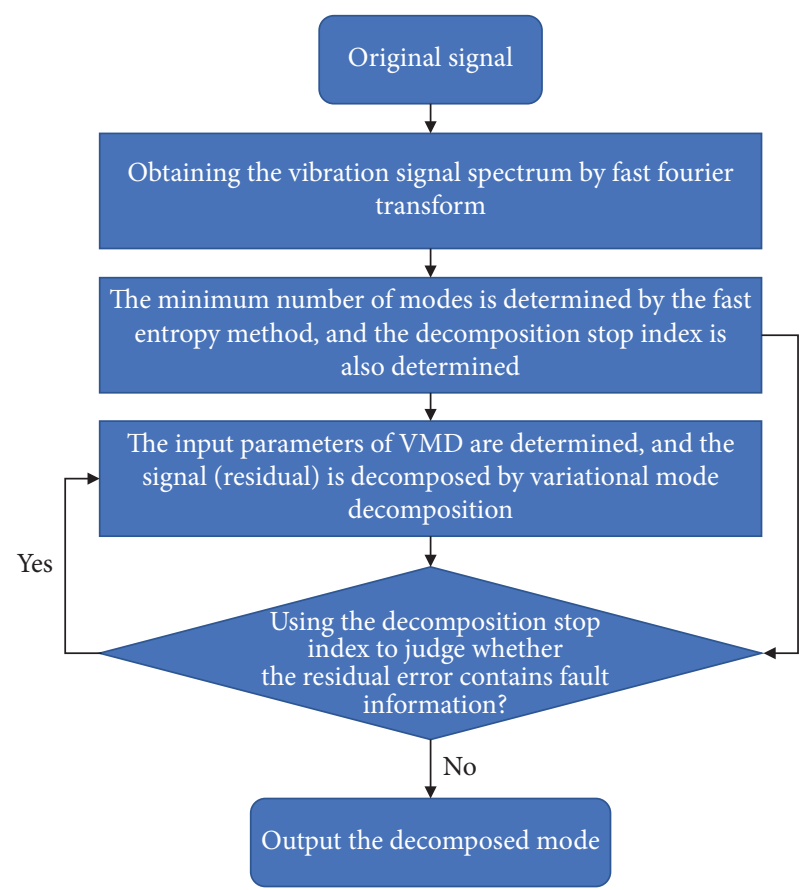

FIgURE 1: Flowchart of the FE-MVMD method.

The calculation method of the proposed fast entropy multilevel variational mode decomposition method is as follows.

Firstly, the number of modes in the original signal is determined by the trend spectrum calculation method in fast entropy, and the frequency spectrum of the initial signal $f(t)$ is obtained by short-time Fourier transform:

$$
\begin{aligned}
F(\omega) & =F[f(t)] \\
& =\int_{-\infty}^{+\infty} f(t) e^{-j \omega t} \mathrm{~d} t .
\end{aligned}
$$

When the signal is processed by using the computer, the continuous signal is discretized:

$$
F(u)=\sum_{n=0}^{L-1} f(n) e^{-i(2 \pi / L) u n} .
$$

The trend spectrum of the spectrum is calculated:

$$
T(f)=\int_{-\infty}^{+\infty} F(u) e^{i 2 \pi u f} \mathrm{~d} u .
$$

The trend spectrum can show the modal components in the signal. The modal components in the signal can be preliminarily determined by extracting the spectral peaks in the trend spectrum, so as to provide the modal number parameters for VMD. The number of qualified modes in the trend spectrum can be obtained by a high-pass filter function:

$$
T(f) * \frac{G_{0}}{1-j\left(\omega_{c} / \omega\right)} \longrightarrow K .
$$

It can be seen from Figure 2 that the threshold value of the filter corresponding to the signal can be determined by fast entropy, that is, the threshold value of the high-pass

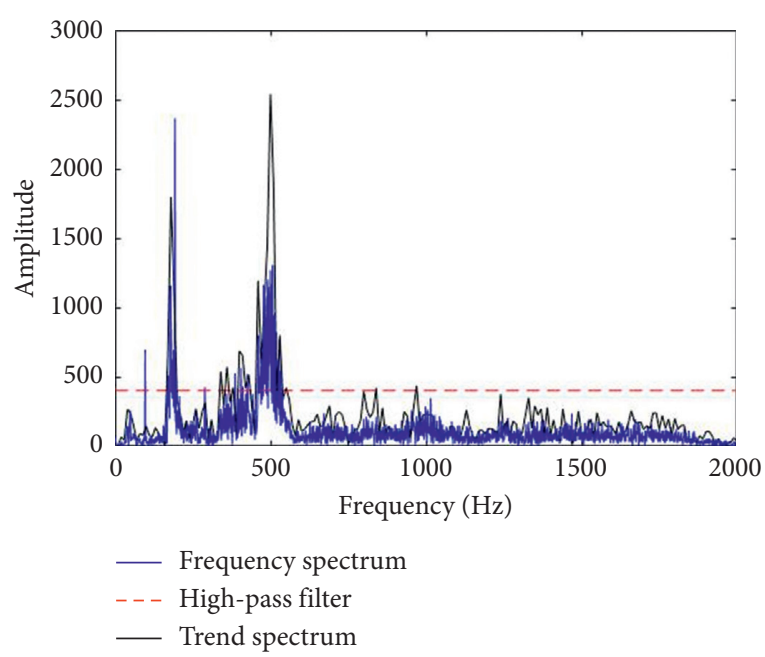

Figure 2: The selection of the signal trend spectrum and modal number.

filter. It can be seen from Figure 2 that the selected threshold value passes through several peaks of the trend spectrum, and the corresponding number of passes can be used as the number of modes used in the multilevel VMD method.

A set of experimental signals are used as samples, and the trend spectrum of the signals is shown in Figure 2.

Figure 2 shows the spectrum of the signal, the trend spectrum of the spectrum, and the high-pass filter obtained by fast entropy calculation. The spectrum is the Fourier spectrum of the signal, and the trend spectrum is used to determine the possible modal components in the spectrum. The modal components in the trend spectrum can be screened by calculating the fast entropy of the spectrum, so as to provide the modal parameter $K$ for the calculation of VMD.

Then, another key input parameter of VMD, the quadratic penalty term $\alpha$, is determined. By analyzing the decomposition results of the signal under different parameters, it is found that the value of $\alpha$ is related to the frequency band size and the center frequency of the extracted modal components, and different values of $\alpha$ correspond to different frequency band sizes. Therefore, the size of the penalty factor determines whether the different frequency bands of the complex signal can be extracted correctly. Therefore, it is necessary to extract the modal components corresponding to different penalty coefficients. The size of the frequency band components can be determined by the slice function in the fast entropy method, and the corresponding penalty coefficients can be obtained:

$$
\omega_{k}^{n+1}=\frac{\int_{0}^{\infty} \omega\left|\widehat{u}_{k}(\omega)\right|^{2} \mathrm{~d} \omega}{\int_{0}^{\infty}\left|\widehat{u}_{k}(\omega)\right|^{2} \mathrm{~d} \omega} \longrightarrow k \alpha .
$$

After obtaining the parameters of VMD, the signal is decomposed into modes. Corresponding to different frequency bands, different parameters are input for iteration, and VMD is performed for many times to obtain the highprecision mode, which is helpful for further analysis of signal components and finding the fault. 


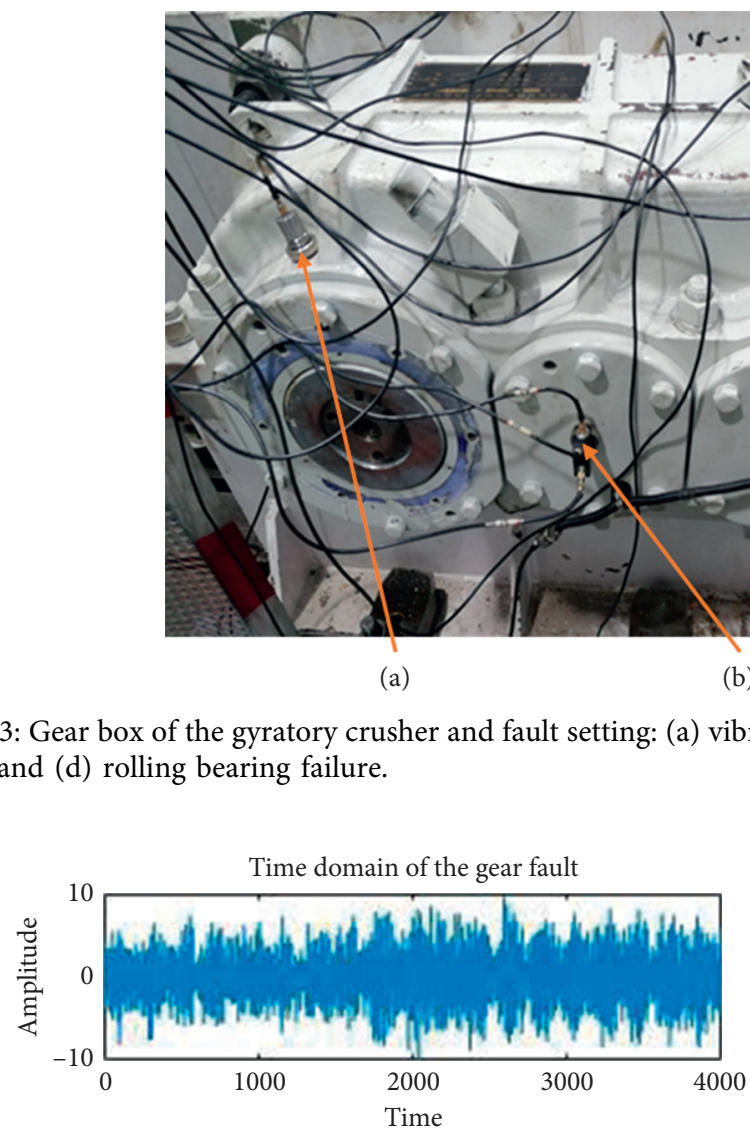

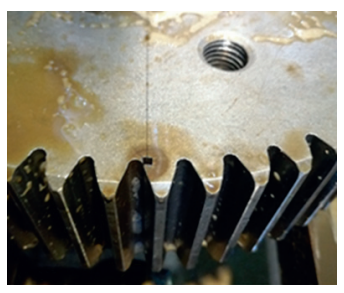

(c)

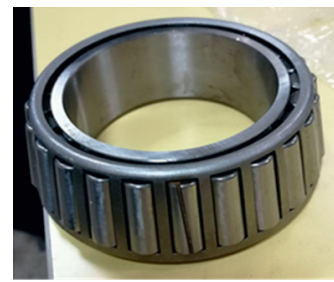

(d)

FIgURe 3: Gear box of the gyratory crusher and fault setting: (a) vibration signal acquisition sensor, (b) speed/acceleration sensor, (c) gear failure, and (d) rolling bearing failure.
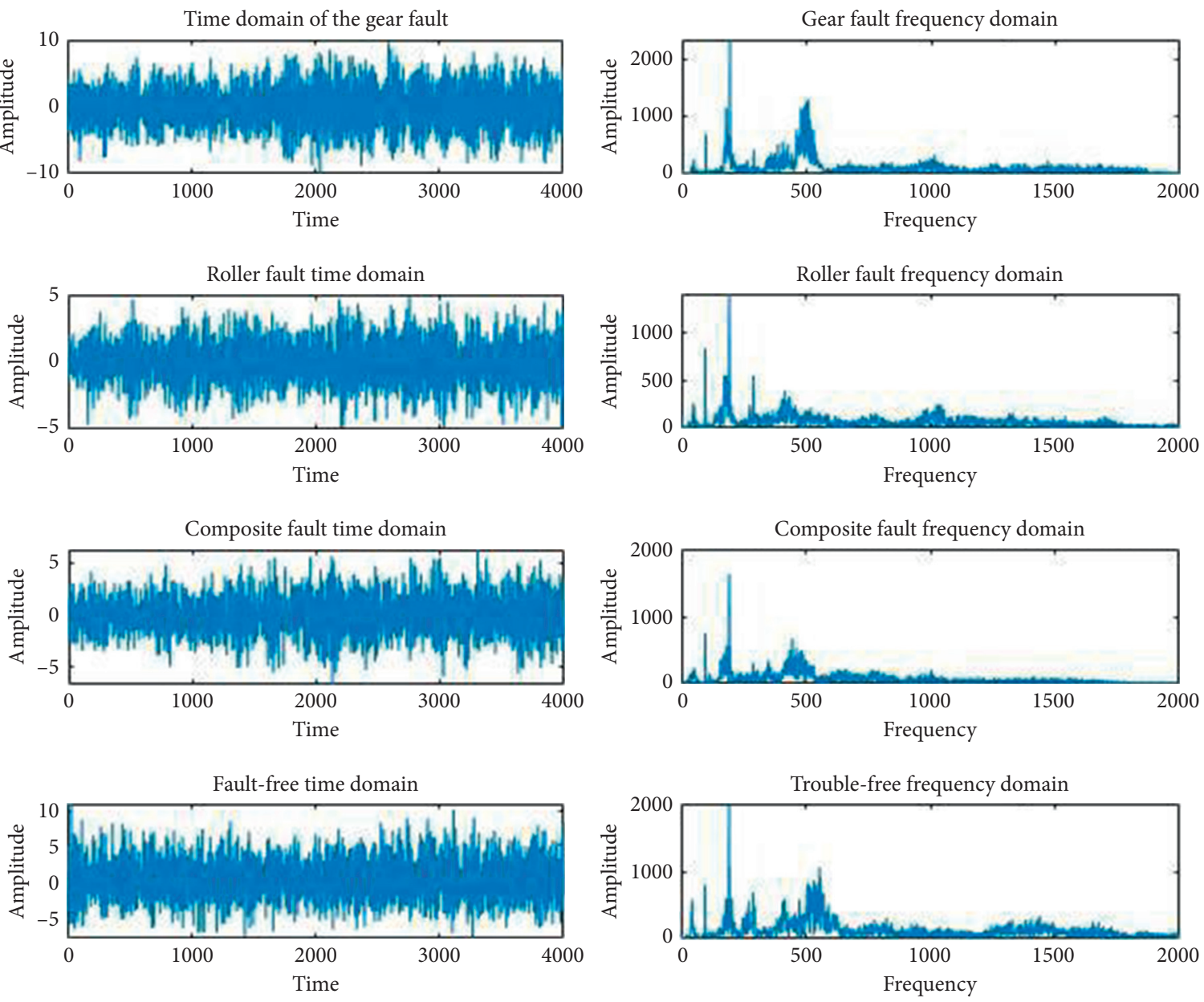

Figure 4: Time-frequency diagram of different faults. 

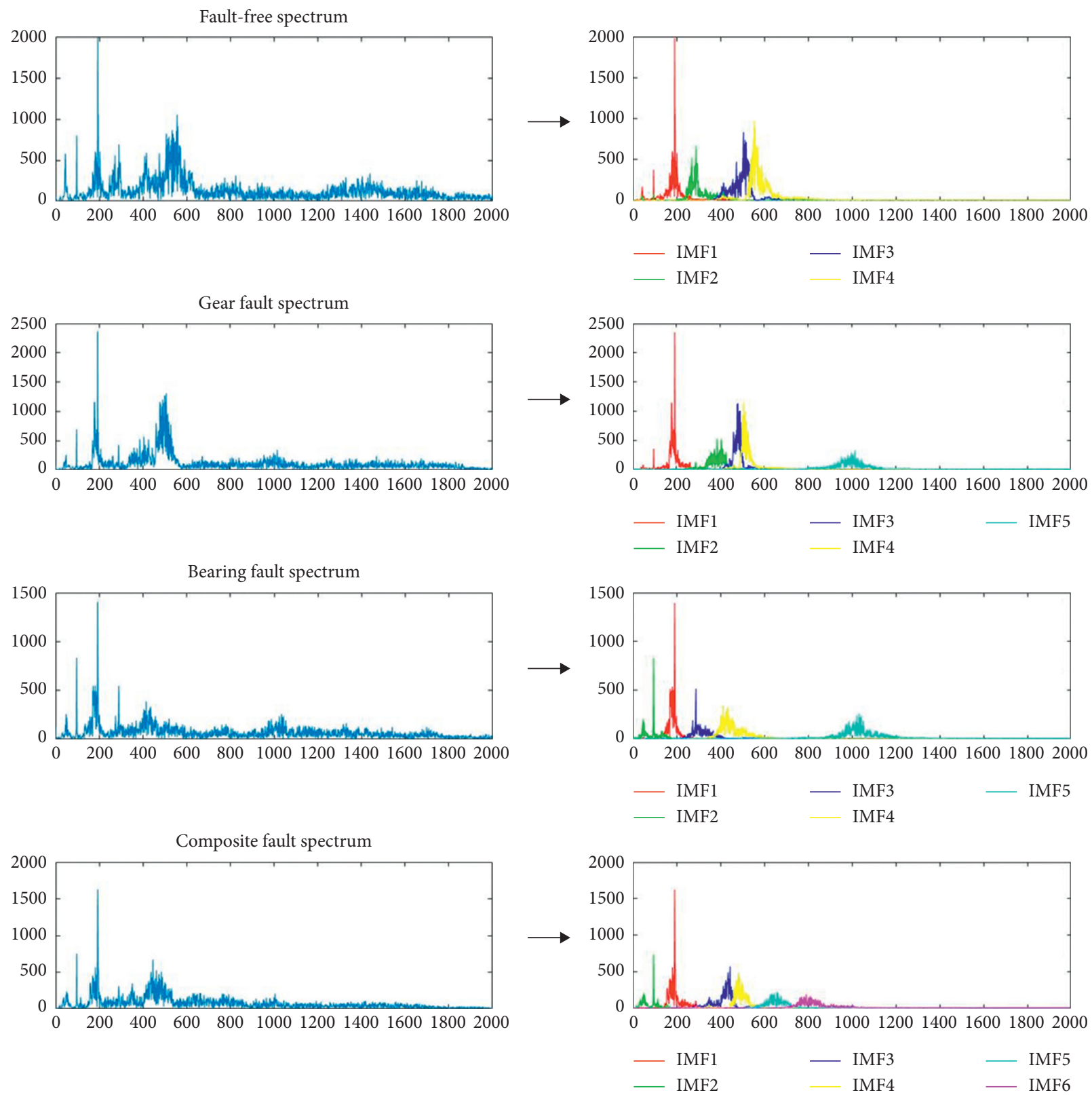

Figure 5: Decomposition results of FE-MVMD.

\section{Experiment and Analysis: Rotating Fault Diagnosis Experiment of the Cycle Crusher}

Gyratory crusher is a kind of rotating machinery commonly used in crushing life line and sand-making life line. Its faults are mostly caused by rotating parts, such as gear and rolling bearing faults. If the rotating faults are unable to be found and eliminated in time, it easily causes safety accidents. In order to solve this problem, it is necessary to analyze the faults of the gyratory crusher. In this paper, the crusher gearbox mechanism is used for data acquisition, and the experimental platform is shown in Figure 3.

Four groups of different types of faults are set in the experiment, which are gear fault, rolling bearing fault, compound fault, and no fault. The bearing type used in the experiment is 33116 bearing, and the gear type is 2TJ06 gear. The sampling frequency used in the experiment is $2048 \mathrm{~Hz}$. Figure 4 shows the time-frequency diagram of the experimental data.

The proposed FE-MVMD method is used to process the four types of data, and the decomposition results are shown in Figure 5.

Through the decomposition results in Figure 5, the ability of the proposed FE-MVMD method to extract signal modes under complex conditions can be verified. Secondly, by comparing the spectrum of the signal before and after decomposition, it can be found that the components of the signal spectrum are effectively put 


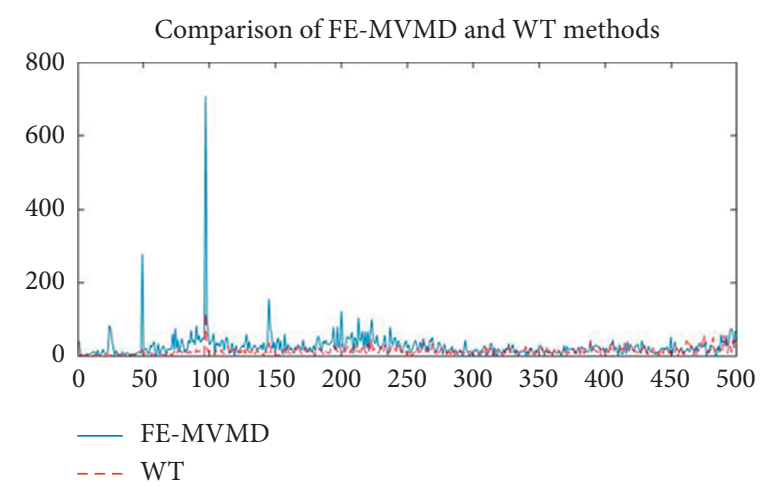

(a)

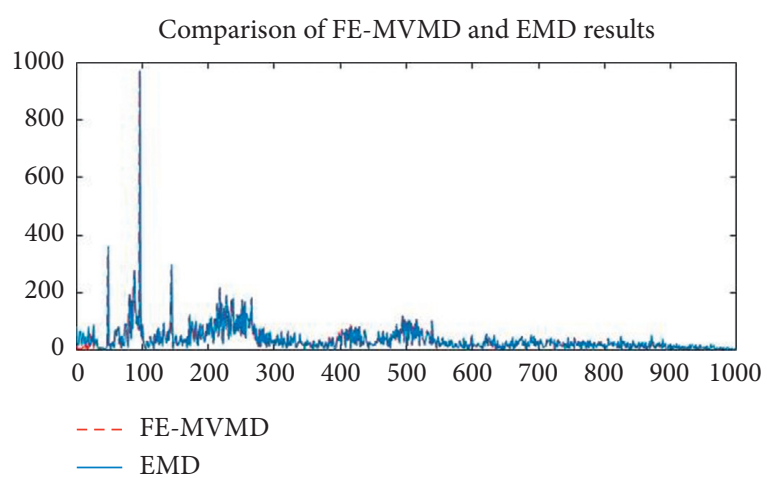

(b)

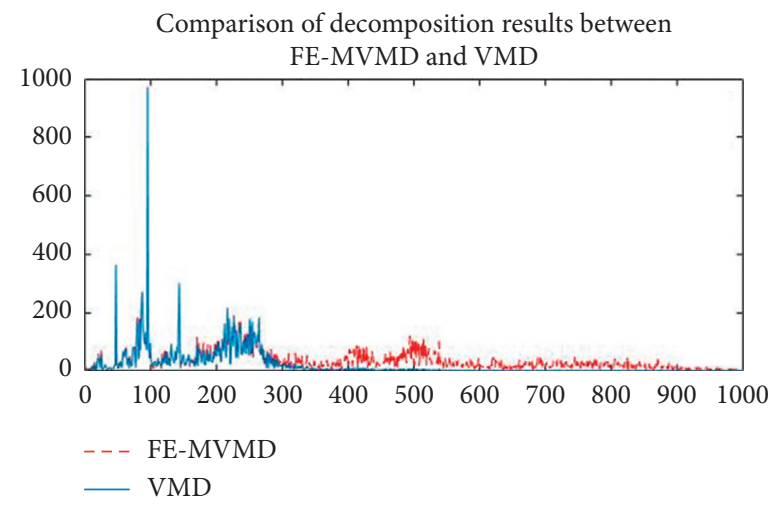

(c)

FIgURE 6: Comparison of modal component extraction results.

TABLE 1: XGBoost modal result classification.

\begin{tabular}{lcccccc}
\hline \multirow{2}{*}{ Methods } & \multicolumn{2}{c}{ Sample } & & \multicolumn{2}{c}{ Result } \\
& Training set & Test set & Gear failure & Bearing failure & Compound fault & No fault \\
\hline WT & 50 & 250 & 0.63 & 0.71 & 0.58 & 0.66 \\
EMD & 50 & 250 & 0.92 & 0.94 & 0.99 \\
VMD & 50 & 250 & 0.98 & 0.97 & 0.95 \\
FE-MVMD & 50 & 250 & 0.98 & 0.99 & 0.97 \\
\hline
\end{tabular}

TABLE 2: XGBoost and RF classification of FE-MVMD features.

\begin{tabular}{lcccccc}
\hline \multirow{2}{*}{ Methods } & \multicolumn{2}{c}{ Sample } & \multicolumn{2}{c}{ Result } \\
& Training set & Test set & Gear failure & Bearing failure & Compound fault & No fault \\
\hline RF & 50 & 200 & 0.95 & 0.97 & 0.92 & 0.99 \\
XGBoost & 50 & 200 & 0.98 & 0.99 & 0.97 \\
\hline
\end{tabular}

forward. Through the comparison between the decomposition results, different modes existing in different faults can be obviously compared. Based on different central frequencies and frequency bands of different modes, the signal components can be judged. In addition, due to the use of fast entropy as the parameter determination method, the optimized parameters are also conducive to filtering out the noise components in the signal, thus improving the reliability of the extraction results of the FE-MVMD method.
In order to further verify the performance of the proposed method, empirical mode decomposition (EMD) and variational mode decomposition (VMD) are used to compare the performance of FE-MVMD. In Figure 6, the decomposition results of VMD are compared with those of FE-MVMD. The number of modes of the VMD method is consistent with that of the MVMD method, so as to highlight the performance of the proposed method. In addition, in order to verify the function of the extracted modal components in fault diagnosis, 200 groups of signals 
under the same fault condition are used as samples to extract the modal components, and the modal components extracted by different methods are trained. XGBoost is used as a classifier, and empirical mode decomposition and variational mode decomposition are used as a comparison to achieve high-accuracy results; the advantages of the FEMVMD method in fault diagnosis were verified.

The experimental signal is the vibration signal generated by the transmission mechanism of the rotary crusher under the actual working condition, and the experimental data contain complex environmental noise. Therefore, the experimental verification using this group of signals can illustrate the antinoise performance of the proposed method. Comparing the decomposition results of FE-MVMD and $\mathrm{VMD}$, it can be found that even if the same number of modes is used as the input, the VMD method is unable to completely separate the modes in the signal. Through Figure 5, it can be seen that the decomposition mode of VMD is concentrated in the low-frequency part, which indicates that the VMD method is unable to find the best band adaptively, so the decomposition effect is not ideal, and the separation of FE-MVMD and EMD is unable to achieve. Comparing the decomposition results of FE-MVMD and the WT method, we can see that the WT method can hardly effectively extract the components in the spectrum. The results of the solution are compared. EMD adaptively decomposes the signal into several modes and a residual. In order to compare the actual effect, the first $K$ modes ( $K$ is the modal quantity parameter adopted by FE-MVMD) are superimposed and compared with the results of FE-MVMD. Through Figure 6, it is observed that the difference between the mode obtained by EMD and FE-MVMD is relatively small, which indicates that the EMD has poor noise resistance and low decomposition efficiency. In summary, the FE-MVMD method proposed to extract the mode is not ideal. The speed and accuracy are better than EMD and VMD algorithms.

In order to verify the actual effect of the FE-MVMD mode in fault diagnosis, it is necessary to use the extracted mode for the training test to verify the classification effect of the extracted mode. In this paper, the XGBoost classifier is used to verify the effect of modal extraction. 50 groups of vibration signals of different fault types are sampled as the training set, and the eigenmode is extracted to form the discrimination basis. In order to verify the classification performance of the proposed method and the effectiveness of modal classification, 250 groups of test sets are used to extract the mode. The results are shown in Table 1.

In order to verify the accuracy and effectiveness of XGBoost, RF (random forest) classification algorithm is added to classify the extracted signal features, which verifies the effectiveness of the proposed method (Table 2).

The classification results show that the classification accuracy of FE-MVMD is better than that of EMD and VMD in different fault conditions, which verifies that FE-MVMD is a kind of signal analysis method suitable for rotating machinery fault diagnosis. The experimental results also show that the classification accuracy of the FE-MVMD method decreases when the modal components are complex, so it is necessary to perform further research to improve the decomposition performance of the FE-MVMD method.

\section{Conclusion}

In this paper, a fast entropy-based multilevel variational mode decomposition (FE-MVMD) method is proposed by combining the basic principles of fast entropy and variational mode decomposition. In this paper, the rotating machinery structure of the gyratory crusher with complex working conditions is taken as the research object, the vibration signal of the mechanical system collected is taken as the analysis sample and compared with other modal extraction methods, and the superior performance of FEMVMD in the signal modal extraction method is verified. In addition, the extracted modal components are used as samples for further fault classification test. XGBoost is used as a classifier to verify the advanced nature of the proposed method in rotating machinery fault diagnosis. In addition, through the experimental process, some conclusions are obtained:

(1) The empirical VMD method usually uses the same parameters to extract the components of the signal, but the actual extraction effect is not ideal.

(2) Through the results of modal extraction, it is found that the influence of noise on the extraction of modal components is very important. Therefore, a reasonable signal denoising method will be beneficial to the effect of modal component extraction.

(3) Based on the process of changing parameters in the proposed MVMD method, a method suitable for local component extraction can be considered, which can greatly improve the efficiency of fault diagnosis.

\section{Data Availability}

The data used to support the findings of this study have not been made available because they are confidential; therefore, they cannot be published.

\section{Conflicts of Interest}

The authors declare no conflicts of interest.

\section{Authors' Contributions}

Fengbiao $\mathrm{Wu}$ and Lifeng Ma conceived and designed the experiments. Guanghui Zhao and Pengtao Liu performed the experiments. Fengbiao $\mathrm{Wu}$ and Qianqian Zhang wrote the paper. All authors read and approved the final manuscript.

\section{Acknowledgments}

This work was supported by the Major Special Projects of Department of Science and Technology of Shanxi Province (Grant no. 20181101017). 


\section{References}

[1] X. Li, W. Zhang, Q. Ding, and J. Q. Sun, "Intelligent rotating machinery fault diagnosis based on deep learning using data augmentation," Journal of Intelligent Manufacturing, vol. 31, no. 2, pp. 433-452, 2020.

[2] W. Teng, X. Ding, S. Y. Tang, J. Xu, and B. S. Shi, "Vibration analysis for fault detection of wind turbine drivetrains-a comprehensive investigation," Sensors-Basel, vol. 21, no. 5, 2021.

[3] Z. Wang, J. Zhou, Y. Lei, and W. Du, "Bearing fault diagnosis method based on adaptive maximum cyclostationarity blind deconvolution," Mechanical Systems and Signal Processing, vol. 162, 2021.

[4] Y. Tan, L. Guo, H. Gao, and L. Zhang, "Deep coupled joint distribution adaptation network: a method for intelligent fault diagnosis between artificial and real damages," IEEE Transactions on Instrumentation and Measurement, vol. 70, pp. 112, 2021

[5] Z. Wang and N. Yang, "A new fault diagnosis method based on adaptive spectrum mode extraction," Structural Health Monitoring, Article ID 147592172098694, 2021.

[6] M. S. Al-Musaylh, K. Al-Daffaie, and R. Prasad, "Gas consumption demand forecasting with empirical wavelet transform based machine learning model: a case study," International Journal of Energy Research, 2021.

[7] C. G. Huang, J. H. Lin, J. M. Ding, and Y. Huang, "A novel wheelset bearing fault diagnosis method integrated CEEMDAN, periodic segment matrix, and SVD," Shock and Vibration, vol. 2018, Article ID 1382726, 18 pages, 2018.

[8] J. Antoni, "Fast computation of the kurtogram for the detection of transient faults," Mechanical Systems and Signal Processing, vol. 21, no. 1, pp. 108-124, 2007.

[9] K. Zhang, Y. G. Xu, Z. Q. Liao, L. Y. Song, and P. Chen, “A novel fast entrogram and its applications in rolling bearing fault diagnosis," Mechanical Systems and Signal Processing, vol. 154, 2021.

[10] S. W. Fei, "fault diagnosis of bearing under varying load conditions by utilizing composite features self-adaptive reduction-based RVM classifier," Journal of Vibration Engineering \& Technologies, vol. 5, no. 3, pp. 269-276, 2017.

[11] J. Zhang, S. Yi, L. Guo, H. Gao, H. Xin, and S. Hongliang, "A new bearing fault diagnosis method based on modified convolutional neural networks," Chinese Journal of Aeronautics, vol. 33, no. 2, pp. 439-447, 2019.

[12] Z. J. Wang and W. H. Du, "Research and application of improved adaptive MOMEDA fault diagnosis method," Measurement, vol. 140, pp. 63-75, 2019.

[13] W. P. He, B. L. Guo, B. Q. Chen, J. J. Ye, and E. Bechhoefer, “A data-driven group-sparse feature extraction method for fault detection of wind turbine transmission system," Measurement Science and Technology, vol. 31, no. 7, 2020.

[14] S. T. Zhao, K. Chang, E. X. Wang, B. Li, K. D. Wang, and Q. Q. Wu, "fault diagnosis of oil pumping machine retarder based on sound texture-vibration entropy characteristics and gray wolf optimization-support vector machine," Shock and Vibration, vol. 2020, Article ID 2709384, 13 pages, 2020.

[15] J. K. Ding, D. M. Xiao, and X. J. Li, "gear fault diagnosis based on genetic mutation particle swarm optimization VMD and probabilistic neural network algorithm," IEEE Access, vol. 8, Article ID 18456, 2020.

[16] L. S. Liu, L. Q. Chen, Z. L. Wang, and D. T. Liu, "Early fault detection of planetary gearbox based on acoustic emission and improved variational mode decomposition," IEEE Sensors Journal, vol. 21, no. 2, pp. 1735-1745, 2021.

[17] D. M. Xiao, J. K. Ding, X. J. Li, and L. P. Huang, "gear fault diagnosis based on kurtosis criterion VMD and SOM neural network," Applied Sciences -Basel, vol. 9, no. 24, 2019.

[18] H. C. Cheng, Y. M. Zhang, W. J. Lu, and Z. Yang, "A bearing fault diagnosis method based on VMD-SVD and fuzzy clustering," International Journal of Pattern Recognition and Artificial Intelligence, vol. 33, no. 12, 2019.

[19] X. M. Xue, C. S. Li, S. Q. Cao, J. C. Sun, and L. Y. Liu, "Fault diagnosis of rolling element bearings with a two-step scheme based on permutation entropy and random forests," EntropySwitzerland, vol. 21, no. 1, 2019.

[20] Q. Li and S. Y. Liang, "An improved sparse regularization method for weak fault diagnosis of rotating machinery based upon acceleration signals," IEEE Sensors Journal, vol. 18, no. 16, pp. 6693-6705, 2018.

[21] K. Elangovan, Y. K. Tamilselvam, M. R. Elara, M. Iwase, T. Nemoto, and K. Wood, "fault diagnosis of a reconfigurable crawling-rolling robot based on support vector machines," Applied Sciences-Basel, vol. 7, no. 10, 2017.

[22] Z. J. Wang, W. L. Zhao, W. H. Du et al., "Data-driven fault diagnosis method based on the conversion of erosion operation signals into images and convolutional neural network," Process Safety and Environmental Protection, vol. 149, no. 12, pp. 591-601, 2021.

[23] M. Azamfar, J. Singh, I. Bravo-Imaz, and J. Lee, "Multisensor data fusion for gearbox fault diagnosis using 2-D convolutional neural network and motor current signature analysis," Mechanical Systems and Signal Processing, vol. 144, 2020.

[24] U. Saeed, S. U. Jan, Y. D. Lee, and I. Koo, "Fault diagnosis based on extremely randomized trees in wireless sensor networks," Reliability Engineering \& System Safety, vol. 205, 2021.

[25] R. Guo, Z. Q. Zhao, T. Wang, G. H. Liu, J. Y. Zhao, and D. R. Gao, "Degradation state recognition of piston pump based on ICEEMDAN and XGBoost," Applied Sciences-Basel, vol. 10 , no. $18,2020$.

[26] K. Dragomiretskiy and D. Zosso, "Variational mode decomposition," IEEE Transactions on Signal Processing, vol. 62, no. 3, pp. 531-544, 2014. 